\title{
HOW TO MEASURE THE CHARM DENSITY IN THE PROTON AT EIC *
}

\author{
N.Ya. IVANOV \\ Yerevan Physics Institute, Alikhanian Bros. Str. 2, 0036 Yerevan, Armenia \\ E-mail: nikiv@yerphi.am \\ www.yerphi.am
}

\begin{abstract}
We study two experimental ways to measure the heavy-quark content of the proton: using the Callan-Gross ratio $R\left(x, Q^{2}\right)=F_{L} / F_{T}$ and/or the azimuthal $\cos (2 \varphi)$ asymmetry in deep inelastic lepton-nucleon scattering. Our approach is based on the following observations. First, unlike the production cross sections, the ratio $R\left(x, Q^{2}\right)=F_{L} / F_{T}$ and the azimuthal $\cos (2 \varphi)$ asymmetry in heavy-quark leptoproduction are sufficiently stable, both parametricallly and perturbatively, in a wide region of variables $x$ and $Q^{2}$ within the fixed-flavornumber scheme of QCD. Second, both these quantities, $R\left(x, Q^{2}\right)=F_{L} / F_{T}$ and $\cos (2 \varphi)$ asymmetry, are sensitive to resummation of the mass logarithms of the type $\alpha_{s} \ln \left(Q^{2} / m^{2}\right)$ within the variable-flavor-number schemes. These two facts together imply that the heavy-quark densities in the nucleon can, in principle, be determined from high- $Q^{2}$ data on the Callan-Gross ratio and/or the azimuthal asymmetry in heavy-quark leptoproduction. In particular, the charm content of the proton can be measured in future studies at the proposed Large Hadron-Electron (LHeC) and Electron-Ion (EIC) Colliders.
\end{abstract}

Keywords: Perturbative QCD, Heavy-Quark Leptoproduction, Mass Logarithms Resummation, Callan-Gross Ratio, Azimuthal Asymmetry

\section{Introduction}

The notion of the intrinsic charm (IC) content of the proton has been introduced about 30 years ago in Ref. [1]. It was shown that, in the light-cone Fock space picture,${ }^{2}$ it is natural to expect a five-quark state contribution, $|u u d c \bar{c}\rangle$, to the proton wave function. This component can be generated by $g g \rightarrow c \bar{c}$ fluctuations inside the proton where the gluons are coupled to different valence quarks. The original concept of the charm density in the

*Talk given at the Workshop on Exclusive Reactions at High Momentum Transfer (IV), May 18-21, 2010, TJNAF, Newport News, VA and Electron-Ion Collider Collaboration Meeting, July 29-31, 2010, Catholic University of America, Washington, DC 
proton $^{1}$ has nonperturbative nature since a five-quark contribution $|u u d c \bar{c}\rangle$ scales as $1 / m^{2}$ where $m$ is the $c$-quark mass. ${ }^{3}$

In the middle of nineties, another point of view on the charm content of the proton has been proposed in the framework of the variable-flavornumber scheme (VFNS). ${ }^{4,5}$ The VFNS is an approach alternative to the traditional fixed-flavor-number scheme (FFNS) where only light degrees of freedom $(u, d, s$ and $g)$ are considered as active. Within the VFNS, the mass logarithms of the type $\alpha_{s} \ln \left(Q^{2} / m^{2}\right)$ are resummed through the all orders into a heavy quark density which evolves with $Q^{2}$ according to the standard DGLAP $^{6}$ evolution equation. Hence this approach introduces the parton distribution functions (PDFs) for the heavy quarks and changes the number of active flavors by one unit when a heavy quark threshold is crossed. Note also that the charm density arises within the VFNS perturbatively via the $g \rightarrow c \bar{c}$ evolution. Some recent developments concerning the VFNS are presented in Refs. [7-9]. So, the VFNS was introduced to resum the mass logarithms and to improve thus the convergence of original pQCD series.

Presently, both nonperturbative IC and perturbative charm density are widely used for a phenomenological description of available data. (A recent review of the theory and experimental constraints on the charm quark distribution may be found in Ref. [10]). In particular, practically all the recent versions of the CTEQ ${ }^{11-13}$ and $\mathrm{MRST}^{14}$ sets of PDFs are based on the VFN schemes and contain a charm density. At the same time, the key question remains open: How to measure the charm content of the proton? The basic theoretical problem is that radiative corrections to the heavy-flavor production cross sections are large: they increase the leading order (LO) results by approximately a factor of two. Moreover, soft-gluon resummation of the threshold Sudakov logarithms indicates that higher-order contributions can also be substantial. (For reviews, see Refs. $[15,16]$.) On the other hand, perturbative instability leads to a high sensitivity of the theoretical calculations to standard uncertainties in the input QCD parameters: the heavy-quark mass, $m$, the factorization and renormalization scales, $\mu_{F}$ and $\mu_{R}, \Lambda_{\mathrm{QCD}}$ and the PDFs. For this reason, one can only estimate the order of magnitude of the pQCD predictions for charm production cross sections in the entire energy range from the fixed-target experiments ${ }^{17}$ to the RHIC collider. ${ }^{18}$

Since production cross sections are not perturbatively stable, they cannot be a good probe of the charm density in the proton. For this reason, it is of special interest to study those observables that are welldefined in $\mathrm{pQCD}$. Nontrivial examples of such observables were proposed 
in Refs. [19-24], where the azimuthal $\cos (2 \varphi)$ asymmetry and Callan-Gross ratio $R\left(x, Q^{2}\right)=F_{L} / F_{T}$ in heavy quark leptoproduction were analyzed. ${ }^{a}$ It was shown that, contrary to the production cross sections, the azimuthal asymmetry $^{20,22}$ and Callan-Gross ratio ${ }^{24}$ in heavy flavor leptoproduction are stable within the FFNS, both parametrically and perturbatively.

In the present talk, we discuss resummation of the mass logarithms of the type $\alpha_{s} \ln \left(Q^{2} / m^{2}\right)$ in heavy quark leptoproduction: ${ }^{23,26}$

$$
l(\ell)+N(p) \rightarrow l(\ell-q)+Q\left(p_{Q}\right)+X[\bar{Q}]\left(p_{X}\right) .
$$

The cross section of the reaction (1) may be written as

$$
\begin{aligned}
\frac{\mathrm{d}^{3} \sigma_{l N}}{\mathrm{~d} x \mathrm{~d} Q^{2} \mathrm{~d} \varphi} & =\frac{2 \alpha_{e m}^{2}}{Q^{4}} \frac{y^{2}}{1-\varepsilon}\left[F_{T}\left(x, Q^{2}\right)+\varepsilon F_{L}\left(x, Q^{2}\right)\right. \\
& \left.+\varepsilon F_{A}\left(x, Q^{2}\right) \cos 2 \varphi+2 \sqrt{\varepsilon(1+\varepsilon)} F_{I}\left(x, Q^{2}\right) \cos \varphi\right],
\end{aligned}
$$

where $F_{2}\left(x, Q^{2}\right)=2 x\left(F_{T}+F_{L}\right)$ while the quantity $\varepsilon$ measures the degree of the longitudinal polarization of the virtual photon in the Breit frame: ${ }^{27}$ $\varepsilon=\frac{2(1-y)}{1+(1-y)^{2}}$. The quantities $x, y$, and $Q^{2}$ are the usual Bjorken kinematic variables while the azimuth $\varphi$ is defined in Fig. 1.

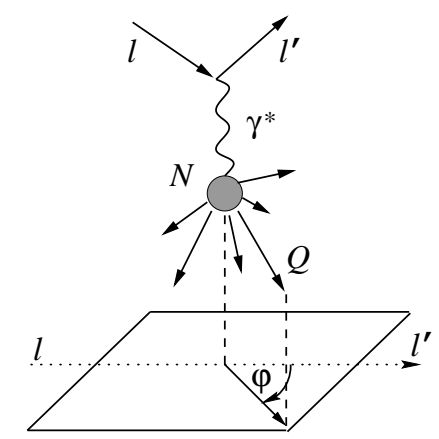

Fig. 1. Definition of the azimuthal angle $\varphi$ in the nucleon rest frame.

In next sections, we will consider resummation of the mass logarithms for the quantities $R\left(x, Q^{2}\right)$ and $A\left(x, Q^{2}\right)$ defined as

$$
R\left(x, Q^{2}\right)=\frac{F_{L}}{F_{T}}\left(x, Q^{2}\right), \quad A\left(x, Q^{2}\right)=2 x \frac{F_{A}}{F_{2}}\left(x, Q^{2}\right) .
$$

\footnotetext{
a Note also the recent paper [25], where the perturbative stability of the QCD predictions for the charge asymmetry in top-quark hadroproduction has been observed.
} 


\section{Resummation for $F_{2}$ and Callan-Gross Ratio}

To estimate the charm-initiated contributions, we use the ACOT $(\chi)$ VFNS proposed in Ref. [7]. ${ }^{\mathrm{b}}$ In Figs. 2 and 3, we present numerical analysis of the NLO corrections ${ }^{28}$ and charm-initiated contributions to the structure function $F_{2}\left(x, Q^{2}\right)$ and Callan-Gross ratio $R\left(x, Q^{2}\right)=F_{L} / F_{T}$ in charm leptoproduction. In our calculations, we use the CTEQ6M parametrization of the gluon and charm PDFs together with the value $m_{c}=1.3 \mathrm{GeV}$ [13]. ${ }^{\mathrm{c}}$ The default value of the factorization and renormalization scales is $\mu=$ $\sqrt{4 m_{c}^{2}+Q^{2}}$.
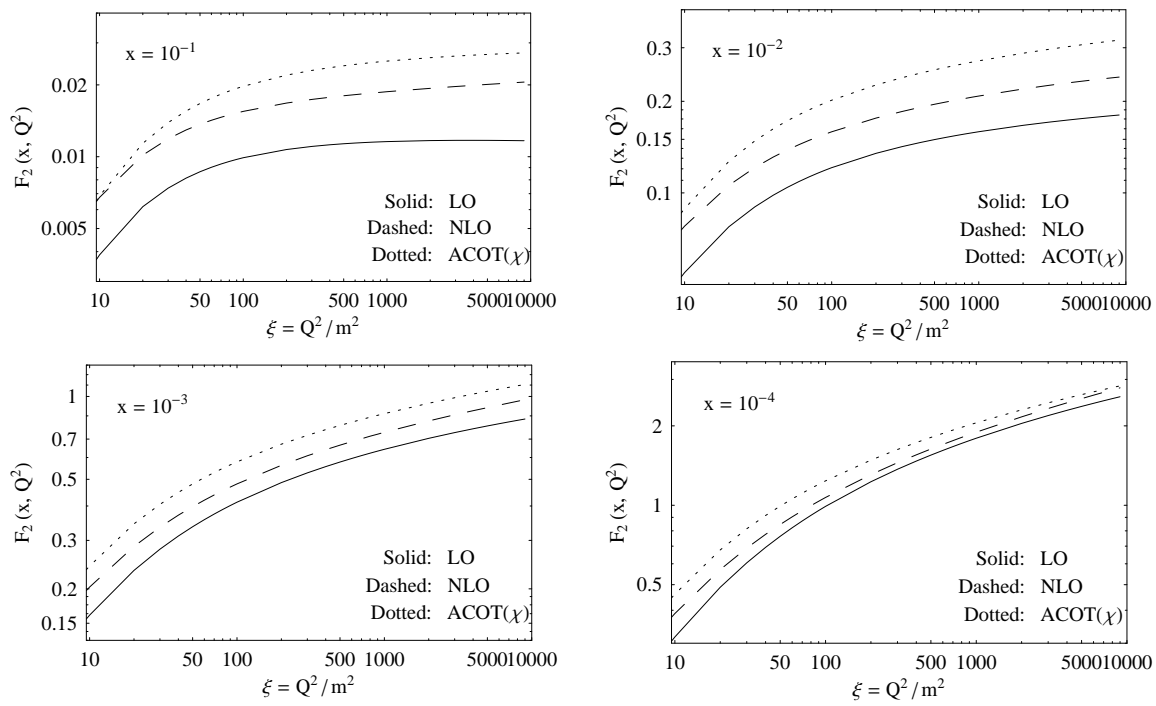

Fig. 2. $Q^{2}$ dependence of the structure function $F_{2}\left(x, Q^{2}\right)$ in charm leptoproduction at $x=10^{-1}, 10^{-2}, 10^{-3}$ and $10^{-4}$. Plotted are the LO (solid lines) and NLO (dashed lines) FFNS predictions, as well as the $\operatorname{ACOT}(\chi) \operatorname{VFNS}$ (dotted curves) results.

One can see from Fig. 2 that, at $x \sim 10^{-1}$, both the radiative corrections and charm-initiated contributions to $F_{2}\left(x, Q^{2}\right)$ are large: they increase the LO FFNS results by approximately a factor of two for all $Q^{2}$. At the same

\footnotetext{
${ }^{\mathrm{b}}$ For more details, see Refs. [23,26].

${ }^{\mathrm{c}}$ Note that we convolve the NLO CTEQ6M distribution functions with both the LO and NLO partonic cross sections that makes it possible to estimate directly the degree of stability of the FFNS predictions under radiative corrections.
} 
time, the relative difference between the dashed and dotted lines does not exceed $25 \%$ for $\xi=Q^{2} / m^{2}<10^{3}$.
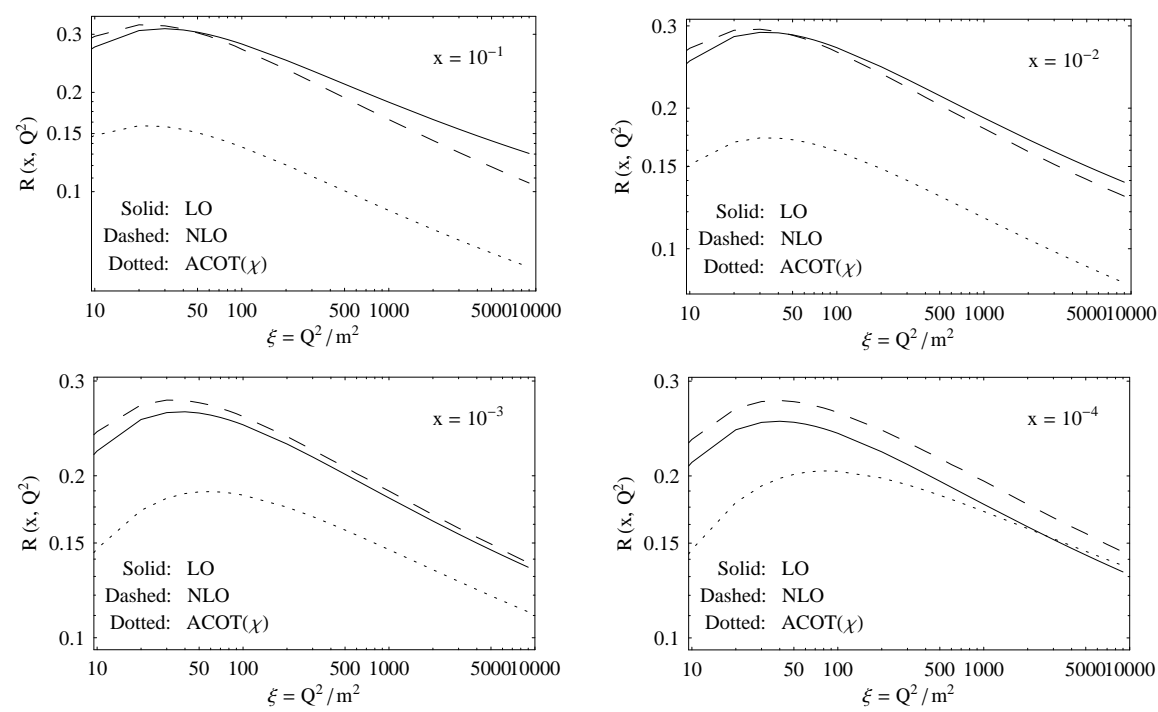

Fig. 3. $Q^{2}$ dependence of the Callan-Gross ratio, $R\left(x, Q^{2}\right)=F_{L} / F_{T}$, in charm leptoproduction at $x=10^{-1}, 10^{-2}, 10^{-3}$ and $10^{-4}$. Plotted are the LO (solid lines) and NLO (dashed lines) FFNS predictions, as well as the $\operatorname{ACOT}(\chi) \operatorname{VFNS}$ (dotted curves) results.

Considering the corresponding predictions for the ratio $R\left(x, Q^{2}\right)$ presented in Fig. 3, we see that, in this case, the NLO and charm-initiated contributions are strongly different. The NLO corrections to $R\left(x, Q^{2}\right)$ are small, less than $15 \%$, for $x \sim 10^{-3}-10^{-1}$ and $\xi<10^{4}$. On the other hand, the corresponding charm-initiated contributions are large: they decrease the LO FFNS predictions by about $50 \%$ practically for all values of $\xi>10$. This is due to the fact that resummation of the mass logarithms has different effects on the structure functions $F_{T}\left(x, Q^{2}\right)$ and $F_{L}\left(x, Q^{2}\right)$ because they have different dependences on the quantities $\alpha_{s}^{n} \ln ^{k}\left(Q^{2} / m^{2}\right)$. In particular, contrary to the transverse structure function, $F_{T}\left(x, Q^{2}\right)$, the longitudinal one, $F_{L}\left(x, Q^{2}\right)$, does not contain potentially large mass logarithms at both $\mathrm{LO}$ and NLO. ${ }^{29,30}$ We conclude that, contrary to the the production cross sections, the Callan-Gross ratio $R\left(x, Q^{2}\right)=F_{L} / F_{T}$ could be good probe of the charm density in the proton at $x \sim 10^{-3}-10^{-1}$.

Note that this observation depends weakly on the PDFs we use. We 
have verified that all the recent CTEQ versions ${ }^{11-13}$ of the PDFs lead to a sizeable reduction of the LO FFNS predictions for the ratio $R\left(x, Q^{2}\right)$.

As to the low $x \rightarrow 0$ behavior of the Callan-Gross ratio, this problem requires resummation of the $\mathrm{BFKL}^{31}$ terms of the type $\ln (1 / x)$ and will be considered in a forthcoming publication.

\section{Resummation for Azimuthal Asymmetry}

Fig. 4 shows the $\operatorname{ACOT}(\chi)$ predictions for the asymmetry parameter $A\left(x, Q^{2}\right)=2 x F_{A} / F_{2}$ at several values of variable $x: x=10^{-1}, 10^{-2}$, $10^{-3}$ and $10^{-4}$. For comparison, we plot also the LO FFNS predictions (solid curves). Again, we use the CTEQ6M parametrization of PDFs, $m_{c}=1.3 \mathrm{GeV}$, and $\mu=\sqrt{4 m_{c}^{2}+Q^{2}}$.
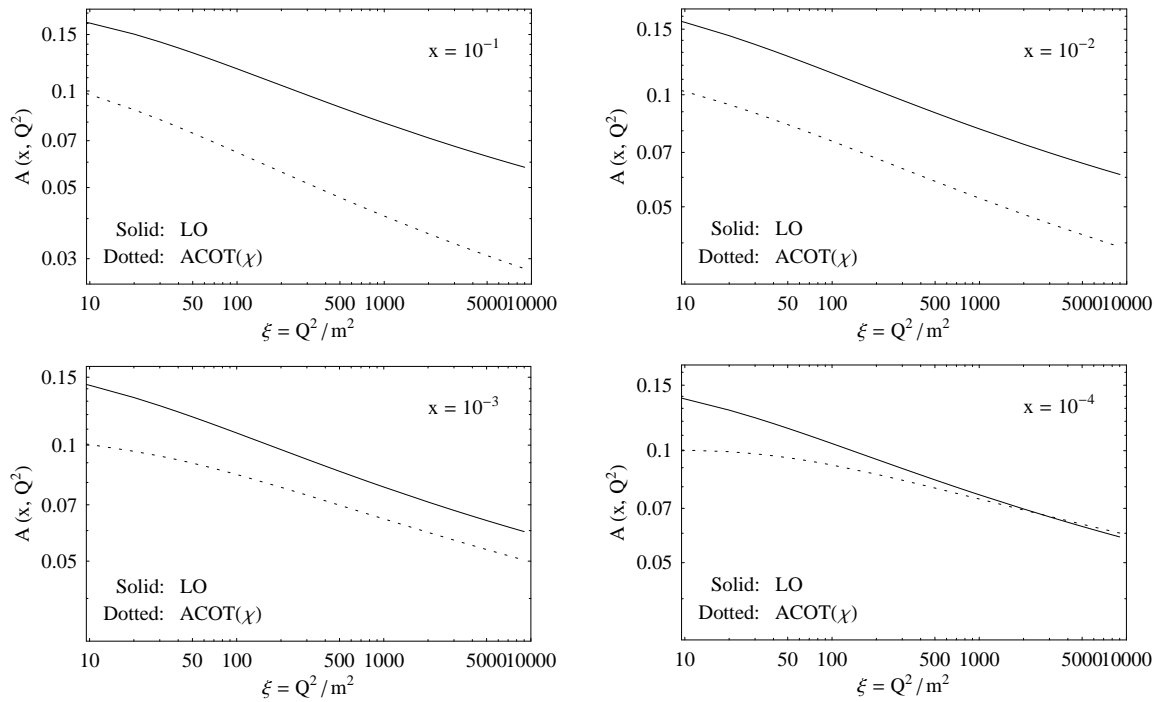

Fig. 4. $Q^{2}$ dependence of the azimuthal asymmetry, $A\left(x, Q^{2}\right)=2 x F_{A} / F_{2}$, in charm leptoproduction at $x=10^{-1}, 10^{-2}, 10^{-3}$ and $10^{-4}$. Plotted are the LO FFNS (solid lines) and $\operatorname{ACOT}(\chi)$ VFNS (dotted curves) results.

One can see from Fig. 4 the following properties of the azimuthal asymmetry. The mass logarithms resummation leads to a sizeable decreasing of the LO FFNS predictions for the $\cos 2 \varphi$-asymmetry. In the $\operatorname{ACOT}(\chi)$ scheme, the charm-initiated contribution reduces the FFNS results for $A\left(x, Q^{2}\right)$ by about $(30-40) \%$ at $x \sim 10^{-2}-10^{-1}$. The origin of this re- 
duction is the same as in the case of $R\left(x, Q^{2}\right)$ : contrary to $F_{2}$, the azimuth dependent structure function $F_{A}$ is safe in the limit $m^{2} \rightarrow 0$ at least at LO.

Presently, the exact NLO predictions for the azimuth dependent structure function $F_{A}$ are not available. However, in Ref. [23] the NLO corrections to the $\cos 2 \varphi$-asymmetry have been estimated within the so-called soft-gluon approximation at $Q^{2} \lesssim m^{2}$. ${ }^{\mathrm{d}}$ It was demonstrated that large soft-gluon corrections to both $F_{A}$ and $F_{2}$ cancel each other in their ratio $A=2 x F_{A} / F_{2}$ with a good accuracy. For this reason, one can exepct that the $\cos 2 \varphi$-asymmetry is also stable, both parametrically and perturbatively, in a wide kinematic range of variables $x$ and $Q^{2}$ within the FFNS.

We have also analyzed how the VFNS predictions depend on the choice of subtraction prescription. In particular, the schemes proposed in Refs. [8,32] have been considered. We have found that, sufficiently above the production threshold, these subtraction prescriptions also reduce the LO FFNS results for the asymmetry by approximately (30-50)\%.

One can conclude that impact of the mass logarithms resummation on the $\cos 2 \varphi$ asymmetry is essential at $x \sim 10^{-2}-10^{-1}$ and therefore can be tested experimentally.

\section{Conclusion}

In the present talk, we compare the structure function $F_{2}$, Callan-Gross ratio $R=F_{L} / F_{T}$ and azimuthal asymmetry $A=2 x F_{A} / F_{2}$ in charm leptoproduction as probes of the charm content of the proton. To estimate the charm-initiated contributions, we used the $\operatorname{ACOT}(\chi) \operatorname{VFNS}^{7}$ and recent CTEQ sets ${ }^{11-13}$ of PDFs. Our analysis of the radiative and charm-initiated corrections indicates that, in a wide kinematic range, both contributions to the structure function $F_{2}\left(x, Q^{2}\right)$ have similar $x$ and $Q^{2}$ behaviors. For this reason, it is difficult to estimate the charm content of the proton using only data on $F_{2}\left(x, Q^{2}\right)$.

The situation with the Callan-Gross ratio and azimuthal asymmetry seems to be more optimistic. Our analysis shows that resummation of the mass logarithms leads to reduction of the FFNS predictions for $R\left(x, Q^{2}\right)$ and $A\left(x, Q^{2}\right)$ by $(30-50) \%$ at $x \sim 10^{-2}-10^{-1}$ and $Q^{2} \gg m^{2}$. Taking into account the perturbative stability of the Callan-Gross ratio and azimuthal asymmetry within the FFNS, ${ }^{23,26}$ this fact implies that the charm density in the proton can, in principle, be determined from high- $Q^{2}$ data on $R=$ $F_{L} / F_{T}$ and $A=2 x F_{A} / F_{2}$.

\footnotetext{
d The soft-gluon approximation is unreliable for high $Q^{2} \gg m^{2}$.
} 
Concerning the experimental aspects, the quantities $R\left(x, Q^{2}\right)$ and $A\left(x, Q^{2}\right)$ in charm leptoproduction can be measured in future studies at the proposed $\mathrm{EIC}^{33}$ and $\mathrm{LHeC}^{34}$ colliders at BNL/JLab and CERN, correspondingly.

\section{Acknowledgments}

We thank S. I. Alekhin and J. Blümlein for providing us with fast $\operatorname{code}^{28}$ for numerical calculations of the NLO DIS cross sections. The author is grateful to H. Avakian, S.J. Brodsky and C. Weiss for useful discussions. This work was supported in part by the ANSEF 2010 grant PS-2033.

\section{References}

1. S. J. Brodsky, P. Hoyer, C. Peterson, and N. Sakai, Phys. Lett. B 93, 451 (1980); S. J. Brodsky, C. Peterson, and N. Sakai, Phys. Rev. D 23, 2745 (1981).

2. S. J. Brodsky, "Light-front QCD" [hep-ph/0412101]; S. J. Brodsky, Few Body Syst. 36, 35 (2005).

3. M. Franz, V. Polyakov, and K. Goeke, Phys. Rev. D 62, 074024 (2000).

4. M. A. G. Aivazis, J. C. Collins, F. I. Olness, and W. -K. Tung, Phys. Rev. D 50, 3102 (1994).

5. J. C. Collins, Phys. Rev. D 58, 094002 (1998).

6. V. N. Gribov and L. N. Lipatov, Sov. J. Nucl. Phys. 15, 438 (1972); Y. L. Dokshitzer, Sov. Phys. JETP 46, 641 (1977); G. Altarelli and G. Parisi, Nucl. Phys. B 126, 298 (1977).

7. W. -K. Tung, S. Kretzer, and C. Schmidt, J. Phys. G 28, 983 (2002).

8. M. Kramer, F. I. Olness, and D. E. Soper, Phys. Rev. D 62, 096007 (2000).

9. R. S. Thorne, Phys. Rev. D 73, 054019 (2006); C. D. White and R. S. Thorne, Phys. Rev. D 74, 014002 (2006); W. K. Tung, H .L. Lai, A. Belyaev, J. Pumplin, D. Stump, and C. -P. Yuan, JHEP 0702, 053 (2007); S. Kretzer, H. L. Lai, F. I. Olness and W. -K. Tung, Phys. Rev. D 69, 114005 (2004); R. S. Thorne and W. K. Tung, [arXiv:0809.0714 [hep-ph]]; P. M. Nadolsky, [arXiv:0809.0945 [hep-ph]].

10. J. Pumplin, Phys. Rev. D 73, 114015 (2006); S. J. Brodsky, B. Kopeliovich, I. Schmidt, and J. Soffer, Phys. Rev. D 73, 113005 (2006); J. Pumplin, H. L. Lai, and W. K. Tung, Phys. Rev. D 75, 054029 (2007).

11. H.L. Lai, J. Huston, S. Kuhlmann, F. Olness, J. Owens, D. Soper, W.K. Tung and H. Weerts, Phys. Rev. D 55, 1280 (1997).

12. H.L. Lai, J. Huston, S. Kuhlmann, J. Morfin, F. Olness, J.F. Owens, J. Pumplin and W.K. Tung, Eur. Phys. J. C 12, 375 (2000).

13. J. Pumplin, D. R. Stump, J. Huston, H. L. Lai, P. Nadolsky, and W. K. Tung, JHEP 0207, 012 (2002).

14. A. D. Martin, R. G. Roberts, W. J. Stirling, and R. S. Thorne, Phys. Lett. B 604, 61 (2004). 
15. E. Laenen and S. -O. Moch, Phys. Rev. D 59, 034027 (1999).

16. N. Kidonakis, Phys. Rev. D 64, 014009 (2001); N. Kidonakis, Phys. Rev. D 73, 034001 (2006).

17. M. L. Mangano, P. Nason, and G. Ridolfi, Nucl. Phys. B 373, 295 (1992); S. Frixione, M. L. Mangano, P. Nason, and G. Ridolfi, Nucl. Phys. B 412, 225 (1994).

18. R. Vogt, Eur. Phys. J. ST 155, 213 (2008).

19. N. Ya. Ivanov, A. Capella, and A. B. Kaidalov, Nucl. Phys. B 586, 382 (2000).

20. N. Ya. Ivanov, Nucl. Phys. B 615, 266 (2001).

21. N. Ya. Ivanov, P. E. Bosted, K. Griffioen, and S. E. Rock, Nucl. Phys. B 650, 271 (2003).

22. N. Ya. Ivanov, Nucl. Phys. B 666, 88 (2003).

23. L. N. Ananikyan and N. Ya. Ivanov, Phys. Rev. D 75, 014010 (2007); L. N. Ananikyan and N. Ya. Ivanov, Nucl. Phys. B 762, 256 (2007).

24. N. Ya. Ivanov and B. A. Kniehl, Eur. Phys. J. C 59, 647 (2009).

25. L. G. Almeida, G. Sterman, and W. Vogelsang, Phys. Rev. D 78, 014008 (2008).

26. N. Ya. Ivanov, Nucl. Phys. B 814, 142 (2009).

27. N. Dombey, Rev. Mod. Phys. 41, 236 (1969).

28. S. I. Alekhin and J. Bluemlein, Phys. Lett. B 594, 299 (2004).

29. E. Laenen, S. Riemersma, J. Smith, and W. L. van Neerven, Nucl. Phys. B 392, 162 (1993).

30. M. Buza, Y. Matiounine, J. Smith, R. Migneron, and W. L. van Neerven, Nucl. Phys. B 472, 611 (1996).

31. E. A. Kuraev, L. N. Lipatov, and V. S. Fadin, Sov. Phys. JETP 44, 443 (1976); I. I. Balitzki and L. N. Lipatov, Sov. J. Nucl. Phys. 28, 822 (1978); L. N. Lipatov, Sov. Phys. JETP 63, 904 (1986).

32. S. Kretzer and I. Schienbein, Phys. Rev. D 58, 094035 (1998).

33. A. Deshpande, R. Milner, R. Venugopalan, and W. Vogelsang, Ann. Rev. Nucl. Part. Sci. 55, 165 (2005).

34. J. B. Dainton, M. Klein, P. Newman, E. Perez, and F. Willeke, J. Inst. 1, P10001 (2006). 